\title{
Multimodal Imitation using Self-learned Sensorimotor Representations
}

\author{
Martina Zambelli* and Yiannis Demiris*
}

\begin{abstract}
Although many tasks intrinsically involve multiple modalities, often only data from a single modality are used to improve complex robots acquisition of new skills. We present a method to equip robots with multimodal learning skills to achieve multimodal imitation on-the-fly on multiple concurrent task spaces, including vision, touch and proprioception, only using self-learned multimodal sensorimotor relations, without the need of solving inverse kinematic problems or explicit analytical models formulation. We evaluate the proposed method on a humanoid iCub robot learning to interact with a piano keyboard and imitating a human demonstration. Since no assumptions are made on the kinematic structure of the robot, the method can be also applied to different robotic platforms.
\end{abstract}

\section{INTRODUCTION}

A vast majority of everyday tasks intrinsically involves feedback from multiple senses and the use of various types of information from different sensory modalities. Multimodal information is then crucial to improve skills and learned self-representations.

Imitation learning methods have been shown effective in enhancing complex robots skills [1,2]. However, although robots are provided with a variety of different sensors, most of the approaches to imitation learning rely on the use of data from a single modality, such as vision $[3,4]$. Solutions for merging different sensors' data have been presented to address object/gesture recognition or speaker identification/spatial localisation [5-7], and more generally, to address classification-type problems, rather than imitating demonstrated behaviours. Studies that explored imitation learning using different sensor information include hierarchical architectures based on multiple internal models [8], Gaussian Mixture Regression together with Hidden Markov Model [9], and reinforcement learning approaches [10]. Motion capture systems (e.g. in [8,9]), as well as kinesthetic and teleoperation are often used with the goal of reproducing a trajectory of the human motion. However these approaches often require manual design of the system and usually a certain number of demonstrations. Bottom-up approaches using motor babbling and self-exploration (e.g. [11,12]) have been studied in the context of imitation learning. These approaches require less prior design while leveraging the advantages of a developmental approach to learning, such as more autonomy, incremental learning and adaptability.

We propose a new method to achieve multimodal imitation on robots, using self-learned sensorimotor representations from visual, proprioceptive and tactile stimuli. The presented method is based on the construction of matrices encoding

${ }^{*}$ M. Zambelli and Y. Demiris are with the Personal Robotics Lab at the Dept. of Electrical and Electronic Engineering, Imperial College London, UK. E-mail: \{m.zambelli13, y.demiris\}@imperial.ac.uk. This work was supported in part by the EU FP7 project WYSIWYD under Grant 612139.

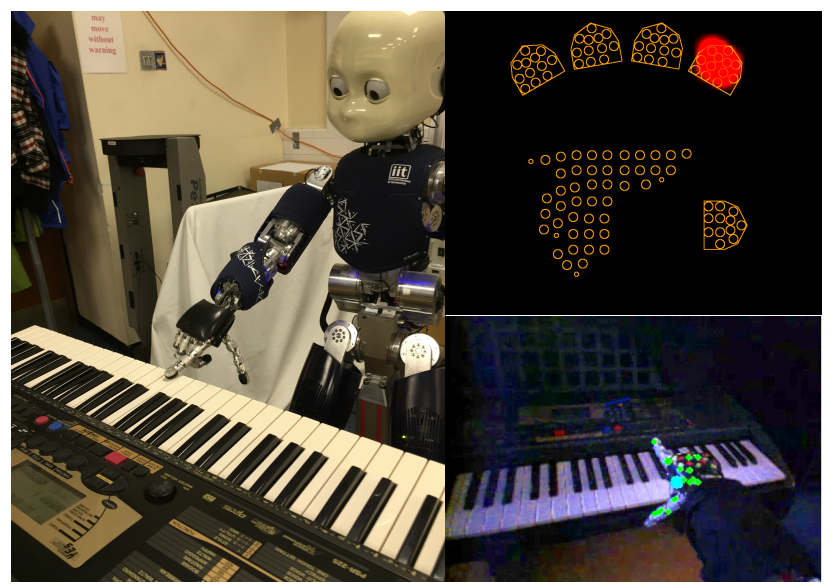

Fig. 1: The iCub robot learns to interact with a piano keyboard through babbling and imitation. The top-right picture shows the hand taxels being activated when keys are pressed. The bottom-right picture shows the view from the robot's left eye camera and the visual features used.

multimodal sensorimotor representations, obtained by juxtaposing unimodal data. The robot then uses the learned representations to calculate on-the-fly new motor commands that are necessary to accomplish multimodal imitation tasks. The proposed framework also allows us to make inference on modalities that are not directly sensed during imitation, using the available sensorimotor information.

Contrary to more classical control approaches based on hand-crafted kinematic and dynamic models, our method allows to achieve multimodal imitation directly on the robot multimodal task space without the need of explicit model formation and without solving inverse kinematics problems. This allows us to avoid the computational burden related to those methods, as well as having the possibility to apply the same method on different robotic platforms.

We tested the proposed method on the task of playing a piano keyboard. A human teacher shows the robot a sequence of notes that the robot should imitate. The demonstration consists of a visual trajectory performed using the same point of view of the robot, although perspective taking [13] could also be employed. No prior knowledge is assumed: the only information available to the robot is the one accumulated during a self-exploration phase in which the robot explores its sensorimotor representations while interacting with the keyboard. To successfully complete the task, the robot must engage concurrently visual and tactile sensory feedback. For evaluation purposes, in order to demonstrate our method also on the proprioception space we further force a constraint on one of the robot's arm joints, so that the overall movement is restricted, thus making the task more difficult for the robot to complete. 
The method is suitable for working with any combination of different modalities, and also is apt to accommodate other modalities. Finally, although we demonstrate our method on a humanoid iCub robot [14], we do not make any assumption on the morphology nor on the kinematics/dynamics of the robot; therefore, the proposed solution can be applied to different robotic platforms.

\section{Methodology}

During a first exploration phase the robot collects information on its sensorimotor capabilities and starts to build relationships between the motor commands issued to the joints and the effect on its sensory systems; this is also known as forward model learning [15,16]. During imitation the robot is able to combine the knowledge previously acquired to track target trajectories defined on its task (perceptive) space, e.g. in visual, tactile and proprioceptive space, in an online manner. Unlike more classical approaches, e.g. [17-19], where the problem of tracking under constraints is based on inverse kinematics and explicit model formulation, our method only relies on self-learned sensorimotor representations and does not require any explicit kinematic model. The proposed method allows the robot to compute the motor commands required to complete the imitation tasks on-the-fly, while the action is taking place.

\section{A. EXPLORATION}

The implementation of our approach is based on a first exploration phase where forward models are learnt. Pseudorandom control signals, referred to as actuation primitives (see also [20]) are issued to the robot's arm joints to generate exploratory movements. In our work, the control signals are velocity commands $v(t)$, defined as function of time for each joint as

$$
v_{j, i}(t)= \begin{cases}v_{j, i} & \text { if } t \in\left[t_{0_{i}}, t_{0_{i}}+d / 2\right) \\ -v_{j, i} & \text { if } t \in\left[t_{0_{i}}+d / 2, t_{0_{i}}+d\right]\end{cases}
$$

where the parameters $v_{j, i}$ and $t_{0_{i}}$ are the magnitude and the starting time of the $i$-th primitive, $i \in\{1,2, \ldots, N\}$, respectively, $j$ indicates the joint number, $j \in\{1,2, \ldots, J\}$, and $d$ is the duration of the primitives. A set of primitives is generated by sampling the magnitudes from a uniform distribution $v_{j, i} \sim \mathcal{U}\left(v_{\min , j}, v_{\max , j}\right)$. Note that the effect of adopting the same duration time for all the primitives is that opposite movements are performed in the first and second part of the primitive execution (see Figure 2). Also, the duration $d$ is kept fixed during the exploration phase, so that the size of each time step considered to build and execute primitives, as well as to acquire and learn sensory effects remains the same during the whole experiment.

During the execution of the randomly generated primitives, data from multiple modalities are acquired: the joints positions from the motor encoders, the position of the hand in the vision field through one of the robot's eye cameras, and the tactile information through the tactile sensors placed on the robot's skin.
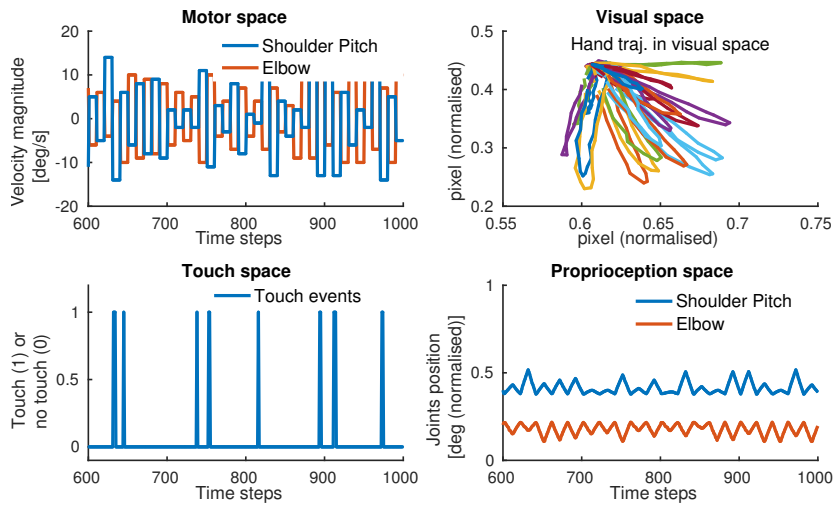

Fig. 2: (Top-left) Velocity command signals for two of the actuators of the iCub arm (representative examples). Trajectories of the (topright) hand positions in the $2 \mathrm{D}$ image space (different colours correspond to different primitives), (bottom-left) touch signal, (bottom-right) proprioception of the shoulder pitch and elbow, during execution of primitives. This figure is best viewed in colour.

Proprioception data: Proprioception information is obtained from the motor encoders attached to each joint of the robot's arm, by recording the positions $q_{1}, \ldots, q_{J}$ (in degrees) of the seven joints of the $\mathrm{iCub}$ arm, and normalising the obtained measures according to each joint's limits. Proprioception information is then collected in a $J \times N$ matrix

$$
\mathrm{S}_{\mathrm{P}}=\left[\begin{array}{ccc}
\Delta_{q_{1}, 1} & \cdots & \Delta_{q_{1}, N} \\
\vdots & \cdots & \vdots \\
\Delta_{q_{J}, 1} & \cdots & \Delta_{q_{J}, N}
\end{array}\right]
$$

where $\Delta_{q_{j}, i}=q_{j}\left(t_{0_{i}}+d / 2\right)-q_{j}\left(t_{0_{i}}\right)$ contains the relative position of joint $j$ between the starting and middle points of execution of primitive $i$.

Vision data: Using the iCub's onboard 2D RGB cameras (with resolution $320 \times 240$ pixel), the position of the hand in the visual space is represented by the $2 \mathrm{D}$ vector $[x, y]^{T}$ of the coordinates of the centre of the hand in the 2D image frames recorded from one of the robot's eye cameras, and normalised according to the frame dimensions. The coordinate of the centre of the hand are computed as the average of the feature points found by using the OpenCV optical flow algorithm. Vision information is collected in a $2 \times N$ matrix

$$
\mathrm{S}_{\mathrm{V}}=\left[\begin{array}{lll}
\Delta_{x, 1} & \cdots & \Delta_{x, N} \\
\Delta_{y, 1} & \cdots & \Delta_{y, N}
\end{array}\right]
$$

where $\Delta_{x, i}=x\left(t_{0_{i}}+d / 2\right)-x\left(t_{0_{i}}\right)$ and $\Delta_{y, i}=y\left(t_{0_{i}}+d / 2\right)-y\left(t_{0_{i}}\right)$ contain the relative displacements of the hand coordinates between the starting and middle points of execution of primitive $i$. An example of randomly generated primitives is reported in Figure 2.

Touch data: Tactile data are recorded through the robot's skin, consisting of a network of tactile sensors (taxels). In our experiments, we are mainly concerned with the hand tactile information. The pressure sensed by the $l$-th taxel of the hand $(l=\{1,2, \ldots, 60\})$ is denoted by $p_{l} \in\{0,1\}$; the average pressure sensed on the palm, calculated as $p=\frac{1}{60} \sum_{l=1}^{60} p_{l}$, is filtered over the duration of the execution of each primitive $i$ to avoid false touch events (e.g. blinking of the sensors), 
binarised and normalised, to obtain $\tilde{p} \in\{0,1\}$. The tactile information is collected in a $1 \times N$ vector

$$
\mathrm{S}_{\mathrm{T}}=\left[\begin{array}{lll}
\Delta_{p, 1} & \cdots & \Delta_{p, N}
\end{array}\right]
$$

where $\Delta_{p, i}=\tilde{p}\left(t_{0_{i}}+d / 2\right)-\tilde{p}\left(t_{0_{i}}\right)$ contains the tactile feedback (ON/OFF) during the execution of primitive $i$.

\section{B. Multimodal Imitation}

The sensorimotor representations gathered during the exploratory phase can then be leveraged to perform imitation tasks. In this section, we introduce our method to combine data from multiple modalities in order to equip the robot with multimodal imitation capabilities.

The formalisation of our method is general and not confined to specific modalities nor robotic platforms. Therefore we define the following notation. Consider $M$ modalities, $m \in\{1,2, \ldots, M\}$, and the multimodal state $s=\left[s_{1}, \ldots, s_{M}\right]^{T}$. We juxtapose multimodal data collected during $N$ self-exploration movements to form a $M \times N$ multimodal sensory matrix

$$
\mathrm{S}=\left[\begin{array}{c}
\mathrm{S}_{1} \\
\vdots \\
\mathrm{S}_{M}
\end{array}\right]=\left[\begin{array}{ccc}
\Delta_{s_{1}, 1} & \cdots & \Delta_{s_{1}, N} \\
\vdots & \cdots & \vdots \\
\Delta_{s_{M}, 1} & \cdots & \Delta_{s_{M}, N}
\end{array}\right],
$$

and the motor commands issued to the joints of the robot's arm used to perform the $N$ self-exploration movements to form a $J \times N$ actuation primitives matrix

$$
\mathrm{A}=\left[\begin{array}{c}
\mathrm{A}_{1} \\
\vdots \\
\mathrm{A}_{J}
\end{array}\right]=\left[\begin{array}{ccc}
v_{1,1} & \cdots & v_{1, N} \\
\vdots & \vdots & \vdots \\
v_{J, 1} & \cdots & v_{J, N}
\end{array}\right] .
$$

Consider now reference trajectories (functions of time) $r_{1}(t), r_{2}(t), \ldots, r_{M}(t)$ that define the target for the imitation task. The imitation error $\varepsilon$, at each time, is the vector of the imitation errors $\varepsilon_{m}=s_{m}(t)-r_{m}(t)$ defined as the distance between the current state $s_{m}(t)$ and the reference $r_{m}(t)$, on each modality space: $\varepsilon(t)=\left[\varepsilon_{1}(t) \cdots \varepsilon_{M}(t)\right]^{T}=\left[\left(s_{1}(t)-r_{1}(t)\right) \cdots\left(s_{M}(t)-r_{M}(t)\right)\right]^{T}$

At each time, the robot moves towards the next reference point, using a combination of the primitives recorded during the exploration phase. The vector of desired velocity commands $v^{*}$ to apply for achieving the multimodal target, denoted as $v^{*}=\left[v_{1}^{*}, v_{2}^{*}, \cdots, v_{J}^{*}\right]^{T}$, is obtained as a combination of the those primitives that led to sensory results which are close to the current target.

We use a range search strategy to find the components of $S$ which are close to the distance $\varepsilon$. In order to optimize the search, a $k$ d-tree model is built from the multimodal sensory matrix $\mathrm{S}$. The $k \mathrm{~d}$-tree algorithm partitions the multimodal sensory matrix $S$ by recursively splitting points in $k$-dimensional space into a binary tree. The $k$ d-tree algorithm allows to optimize the search of neighbours, and it is particularly useful when $k$ is relatively small; in our case $k$ always remain limited, for example if one joint is constrained, and visual and tactile trajectories are defined, $k=4$, while the number of samples in the exploration dataset can always satisfy $N \gg k$. The indices of the columns of $S$ found by the range search are used to select the corresponding columns in the actuation primitives matrix $A$.

We denote by $\tilde{S}$ and $\tilde{A}$ the matrices containing the nearest neighbours columns of $S$ and $A$, respectively. Note that the relative position to the target, given by $\varepsilon$, can be expressed in terms of the relative sensory effects recorded during the exploration phase contained in the matrix $\tilde{\mathrm{S}}$. This relation can be formulated as $\tilde{S} w=\varepsilon$, where $w$ is a weighting vector. The solution of this equation, which defines a least square regression problem, gives the solution for the control problem in the task (sensory) space, and explains the current relative state as a weighted combination of those ones observed during the exploration phase. The best approximate solution, also the minimum norm solution, is given by $w=\tilde{S}^{\dagger} \varepsilon$, where $\tilde{S}^{\dagger}$ denotes the pseudoinverse of the matrix $\tilde{S}$. If $\tilde{S}$ is not full rank, a common approach to obtain a well-defined solution is to compute $\mathrm{w}$ as $\mathrm{w}=V \Sigma^{\dagger} U^{*} \varepsilon$, where $V, \Sigma, U$ are matrices found with the single value decomposition (SVD) of the matrix $S$, and $\Sigma^{\dagger}$ denotes the pseudoinverse of the matrix $\Sigma$. Since each column in $\tilde{S}$ is directly related to a particular primitive, and to a particular column in $\tilde{A}$, the same vector w can be used to relate new primitives with the primitives recorded in the exploration phase, that is $v^{*}=\tilde{A} w$. This equation defines the desired motor command vector as a combination of the nearest primitives previously observed through the weight vector w. Note that the desired motor command vector $v^{*}$ is found without solving any inverse kinematic problem.

Vision, Touch and Proprioception: Consider now the specific cases of vision, touch and proprioception. The imitation error vector is defined as $\varepsilon=\left[\begin{array}{lll}\varepsilon_{\mathrm{P}} & \varepsilon_{\mathrm{V}} & \varepsilon_{\mathrm{T}}\end{array}\right]^{T}$ and $\varepsilon_{\mathrm{P}}, \varepsilon_{\mathrm{V}}, \varepsilon_{\mathrm{T}}$ are defined, respectively, as

$$
\varepsilon_{\mathrm{P}}=\left[q_{n}(t)-q_{n}^{*}(t)\right], \quad \varepsilon_{\mathrm{V}}=\left[\begin{array}{l}
x(t)-x^{*}(t) \\
y(t)-y^{*}(t)
\end{array}\right], \quad \varepsilon_{\mathrm{T}}=\left|\tilde{p}(t)-\tilde{p}^{*}(t)\right|
$$

where the couple $(x(t), y(t))$ represents the current hand position, and the couple $\left(x^{*}(t), y^{*}(t)\right)$ the target position at time $t ; \tilde{p}(t)$ denotes the pressure signal at time $t$, and $\tilde{p}^{*}(t)$ the target contact pressure at time $t ; n$ is the number of the joint (multiple joints can also be considered) on which a constraint (reference) $q_{n}^{*}$ is given. Note that for the touch modality, which is represented by a binary signal, the state and the imitation error are as well binary variables, taking values 0 or 1 .

\section{INFERENCE ON MISSING MODALITIES}

The framework proposed in this paper can also be used to perform inference on missing modalities. As, for example, humans can infer others' tactile sensations only based on visual clues, intelligent robotic systems should be able to infer modalities that can not directly be measured. A context on which inferring missing modalities can be useful is the case of faulty sensors: for example, if the touch sensors fail during the execution of a task, but the robot knows from previous experience that tactile feedback should be perceived, it can act accordingly to compensate or stop and ask external intervention. 
The proposed method allows us to make inference on a missing modality by leveraging the information contained in the multimodal sensory matrix S. More specifically, if the state $s_{m_{1}}$ from modality $m_{1}$ is available, it is possible to infer the state $s_{m_{2}}$ given $s_{m_{1}}$ by performing a nearest neighbours search on $S_{m_{1}}$ to find the column vectors that are closest to the state $s_{m_{1}}$; the corresponding columns can then be extracted from $S_{m_{2}}$. Denoting with $\tilde{\mathrm{S}}_{m_{2}}$ the matrix containing the columns selected from $S_{m_{2}}$, the state $s_{m_{2}}$ can be inferred as a combination of the columns of $\tilde{\mathrm{S}}_{m_{2}}$, that is $\hat{s}_{m_{2}}=\tilde{\mathrm{S}}_{m_{2}} \mathrm{w}$, where $\hat{s}_{m_{2}}$ is the inferred state of modality $m_{2}$ and $\mathrm{w}$ is a $l \times 1$ weighting vector, where $l$ is the number of neighbours found in $S_{m_{1}}$.

\section{EXPERIMENTS AND DISCUSSION}

We have demonstrated our approach on an iCub humanoid robot. The iCub learns to interact with a piano keyboard using vision, touch and proprioception. First the robot executes babbling movements to explore sensorimotor representations (see Figure 1). In order to avoid harmful collisions with the piano keyboard, the random velocities used to perform the exploratory movements have been sampled from biased uniform distributions, bounded by safe minimum and maximum velocities.

After exploration, a demonstrator shows the robot how to play a sequence of notes, by demonstrating a visual trajectory of the hand used to play the piano (see Figure 3). Note that the point of view of the robot and of the teacher during the demonstrated execution is nearly the same, although perspective taking [13] could also be employed. The task assigned to the robot is to imitate the demonstrator execution based on the visual trajectory demonstrated. Touch

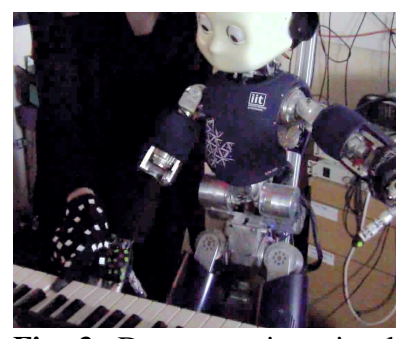

Fig. 3: Demonstrating visual trajectories to the iCub robot. is fundamental in order to successfully play the piano keys, hence the task is multimodal. To make the task more complicated, we also force a constraint on proprioception by fixing one degree of freedom of the arm, so that the robot is forced to execute the imitation task without actually exploiting one of the arm's degrees of freedom. This constraint can also be seen as simulating a faulty joint: the robot is required to complete the task nonetheless, while its operational space is reduced.

We show that the robot is able to leverage the multimodal data acquisition and the self-learned multimodal sensorimotor representations to complete the multimodal imitation task. The juxtaposition used to build the multimodal matrices benefits multimodal imitation tasks by concurrently meeting requirements defined on different sensory spaces.

The demonstrated visual trajectory is described by $N_{\text {target }}=135$ points, acquired during the demonstration through one of the robot's eye cameras. The demonstrated trajectory is the sequence of positions of the teacher hand in the robot's image frames (see Figure 4).
$N_{\text {target }}$ only represents the number of sampled positions defining the demonstrated trajectory, and it is not directly related to the duration $d$ of the exploration primitives, which is only used during the exploration phase.

Regarding the touch modality, as a matter of fact, humans

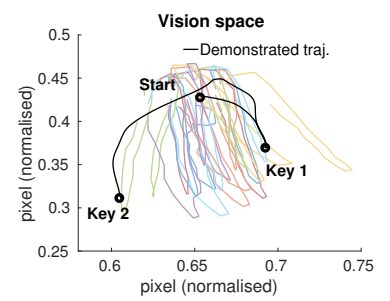

Fig. 4: Visual trajectories: explored (coloured transparent lines), demonstrated (black). can not directly observe tactile sensations from others. On the other hand they are able to infer the tactile sensation for example from visual clues. We show (in Section III-C) that our method can be applied to infer missing modalities, while synthetic trajectories are used for the imitation experiments. More specifically, a piecewise constant reference $\tilde{p}^{*}$ is provided, defined as $\tilde{p}^{*}=1$ when the key should be pressed, and $\tilde{p}^{*}=0$ during the transition movements from the starting position to the first key, and from the first to the second key.

A reference constant trajectory is also synthetically provided only for one joint, namely the shoulder yaw, which is forced to remain fixed. This allows us to simulate a condition when the robot is limited in its motion capabilities, for example by a faulty joint. By providing a constant reference $q_{n}^{*}(t)=$ const for all $t$ and for a specific $n$ (in our case $n=3$ ), we effectively force a constraint in the proprioception space, which in turns limits the robot's operational space. In other works, proprioceptive references (joint angle data), were acquired for example from motion capture systems, e.g. gloves [9], or more elaborate vision processing, e.g. [21], which are beyond the scope of this paper. For the purpose of demonstrating the effectiveness of our method, we let the application of these approaches as future work and input a synthetic target trajectory to force a constraint on one of the robot's degrees of freedom.

\section{A. Multimodal imitation On PiAnO KEyBoARd}

The imitation task considered is difficult for different reasons: first, during the exploration the robot might have touched the keyboard only a few times and only in certain positions of the image frames; second, some positions of the demonstrated trajectory in the visual space might have never been explored before; third, during the exploration the robot learns sensorimotor relationships using more degrees of freedom than the available degrees of freedom in the imitation task, when the effective operation space is reduced by the constraint forced on the proprioception space. Note that performing the imitation task without this constraint would be easier for the robot, since it is forced to solve the task on-the-fly using less degrees of freedom than those available during the exploration phase.

Experimental results show that our method can effectively handle these issues. A key impact to the effectiveness of the method is the search of similar sensory states on the multimodal space, rather than on each single modality.

In Figure 5 the results of the multimodal imitation task are shown, for 10 repetitions of the task. The robot follows 

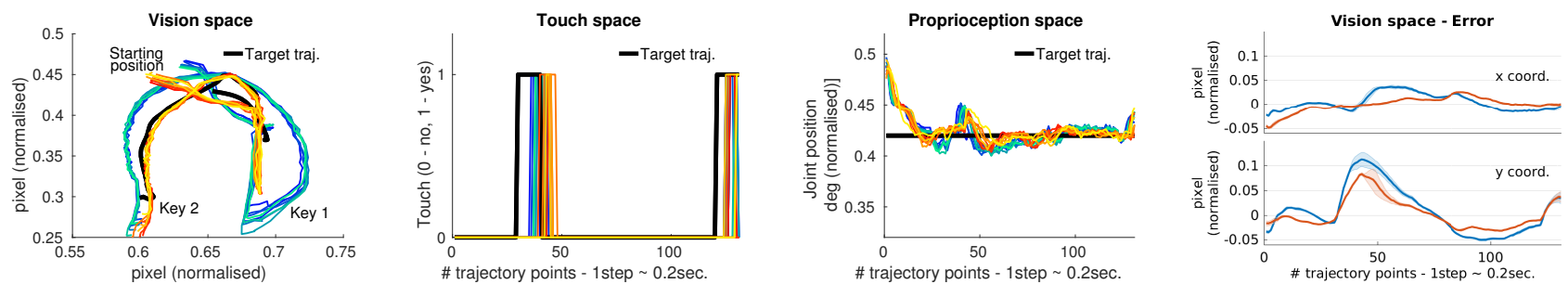

Fig. 5: Multimodal imitation: qualitative results on the vision, touch and proprioception spaces, and temporal profile of the error in the visual space (leftmost figure). Blue lines and orange/yellow lines refer to results obtained using $N=20$ and $N=50$, respectively. The improvement in the performance achieved using more exploration steps can be noted especially in the vision and proprioception cases. All figures show the results of 10 repetitions. This figure is best viewed in colour.

the demonstrated visual trajectory but in order to satisfy the touch modality requirement, that is in order to actually touch the piano keys, the overall behaviour in the visual space is a generalization of the demonstrated trajectory. It can be noted that the most difficult part in the execution of this imitation task happens just after the first key is pressed.

Because the teacher shows a visual demonstration, the experiment is not restricted to a particular set of predefined notes. On the contrary, the demonstration can include any number of keys, at different positions on the keyboard contained in the robot's visual field. Note however that the imitation results depend on the learned representations built in the self-exploration phase: the information regarding the sensorimotor representations, that is the motor effects on the sensory system and on the interaction with the keyboard, is all and only the one collected in the self-exploration phase. Although the accumulated information have a notable impact on the imitation outcome, the proposed method allows to combine previous information to reach also unexplored points in the multimodal space.

We have run 50 repetitions of the full experiment, where the $\mathrm{iCub}$ robot is required to play consequentially two keys, as from the teacher demonstration. The success achieved, that is the successful execution of the two notes, was 45 over 50 $(90 \%)$. The failures were not due to the fact that the keys were not reached but to the fact that the tactile data acquired from the robot's fingertip were sometimes imprecise.

\section{B. INFLUENCE OF PARAMETERS}

The outcomes of the imitation task can be affected by the following design parameters: the number $N_{\text {target }}$ of points that define the target trajectories, the radius $r$ chosen to perform the range search on the $k \mathrm{~d}$-tree, and the number $N$ of samples acquired.

Influence of $N_{\text {target }}$ : The number of points defining the target trajectories has a direct impact on the time taken to complete the imitation task: the more points, the more refined is the trajectory path, the more accurate is the tracking result, the slower the execution. Experimental observations suggest a minimum of $N_{\text {target }}=100$ for a relatively small movement. In our experiments we have used $N_{\text {target }}=135$, corresponding to approximately 30 seconds. Recall that $N_{\text {target }}$ is not directly related to the duration $d$ of the exploration primitives. On the other hand, the number of points $N_{\text {target }}$ collected during the demonstration depends
TABLE I: Effect of $r$ and $N$ on imitation performance (over 10 repetitions). Normalisation is applied to the error measures for better comparison. Results are reported in the form median[25th percentile; 75 th percentile].

\begin{tabular}{llll}
\hline & Proprioc. & Vision & Touch \\
\hline$r=0.25$ & $0.34[0.17 ; 0.40]$ & $0.26[0.12 ; 0.87]$ & $0.35[0.28 ; 0.78]$ \\
$r=0.3$ & $0.59[0.32 ; 0.68]$ & $0.27[0.12 ; 0.34]$ & $0.28[0.21 ; 0.50]$ \\
$r=0.35$ & $0.71[0.41 ; 0.81]$ & $0.37[0.24 ; 0.54]$ & $0.57[0.35 ; 0.71]$ \\
\hline$N=20$ & $0.86[0.76 ; 0.89]$ & $0.86[0.69 ; 0.93]$ & $0.20[0.13 ; 0.40]$ \\
$N=50$ & $0.16[0.09 ; 0.28]$ & $0.08[0.01 ; 0.21]$ & $0.70[0.33 ; 0.73]$ \\
\hline
\end{tabular}

on the sampling rate used to record sensory data (that is, in this work, approximately 5 hertz).

Influence of $r$ : The parameter $r$, denoting the width of the radius used to perform the range search, sets the number of column vectors chosen from $S$ and $A$ at each time. Using the range search, the number of neighbours varies for every query point. The situation in which the robot can not find similar experience from the past exploration leads to a void search, that is no neighbours are found in S. This void search then corresponds to no motor commands being chosen among the column of the matrix A, and the robot would stay still. This behaviour can be seen as a limitation but in fact it reflects directly the influence of the previous experience on the imitation task. In this situation, either a larger $r$ is allowed, or more exploration should be performed. In the first case, samples which are farther from the query can be taken into account; this solution corresponds to allowing the robot to try to combine the (insufficient) accumulated knowledge to reach for the target anyway. In the second case, the robot would stop trying to track the target and will focus on gathering more data from self-exploration, with the aim of collecting a more complete set of samples. However, experimental results show that as long as $r$ is chosen so that there exist neighbours almost all the times, the imitation performance does not improve sensibly (see Table I). The main reason is that the number of neighbours in fact depends on the number of samples collected in the exploration phase and the portion of the multimodal space actually explored (discussed in the next paragraph). From a computational point of view, it can be noted that the higher $r$, the bigger the matrix $\tilde{\mathrm{S}}$. This might potentially cause higher computational complexity, since $\tilde{S}$ must be inverted to find the weight vector w. However, in practice the number of neighbours found will hardly be bigger than 100 units, thus making the inversion of $\tilde{S}$ easily computable. 
Influence of $N$ : The choice of $N$ determines the number of primitives and sensorimotor samples gathered, and potentially how much of the multimodal space is effectively explored. In Table I the results show the performance obtained by using different amount of exploration data, with $N_{\text {target }}=135$, for 10 repetitions. While the results for the tactile space are affected by sensible delays when the first key should be pressed (see qualitative results in Figure 5), it can be noted that a considerable improvement is achieved by increasing the number of primitives used from $N=20$ to $N=50$ especially in the vision and proprioception space. In particular, the movement performed using $N=50$ is more precise, especially when the motion to reach the second key starts. This is in fact a critical moment in the imitation (see also the temporal profile of the vision error in Figures 5), also because of the constraint forced on the proprioception space: note the pick of the proprioception error in correspondence of the pick in the vision space (around time step 50).

\section{INFERENCE ON MISSING MODALITY}

In order to test our method on the capability of performing inference on missing modalities, we assume that the tactile information is no longer available during the imitation task, and we calculate the inferred touch, given the proprioception information. The task now consists of playing only one note, while maintaining a constant contact with the keyboard. Given the binary nature of the tactile data, we have evaluated the percentage of time steps where $\varepsilon_{\mathrm{T}} \neq 0$ during the execution of this task. The percentage scores obtained for the executed and inferred trajectories were $14.8 \%$ and $4 \%$, respectively. Interestingly inference allows to gain valuable insight of the actual experiment: the inference result could allow to overcome physical limitations affecting the actual execution while providing information which can be used to enhance the control policy. For example the inferred modality values could be fed back to the controller to compensate for imitation errors. Also, being based on the same multimodal sensory matrices used for the imitation task, the inference step can be easily integrated and used online during the task execution.

\section{CONCLUSION}

We have presented a method to enhance robots with multimodal learning skills enabling imitation and inference on missing modalities. Our method, based on self-learned multimodal sensorimotor representations, benefits from using data from multiple sensors from self-exploration, from the multimodal sensory matrices representation and from the range search approach on multimodal space. The formulation of the proposed method is also general and allows to accommodate other modalities. Finally, we have demonstrated our method on a humanoid iCub robot. However, since no a priori knowledge has been assumed on the kinematic and dynamic models of the robot, the proposed method can be applied to different robotic platforms.

We are currently working on extending the experimental scenario in order to include the sound in the multimodal space, as well as demonstrating the method on different robots.

\section{REFERENCES}

[1] A. Billard, S. Calinon, R. Dillmann, and S. Schaal, "Robot programming by demonstration," in Springer handbook of robotics. Springer, 2008, pp. 1371-1394.

[2] S. Schaal, A. Ijspeert, and A. Billard, "Computational approaches to motor learning by imitation," Philosophical Transactions of the Royal Society B: Biological Sciences, vol. 358, no. 1431, pp. 537-547, 2003.

[3] Y. Wu and Y. Demiris, "Hierarchical learning approach for one-shot action imitation in humanoid robots," International Conference on Control, Automation, Robotics and Vision, pp. 453-458, 2010.

[4] M. Lopes and J. Santos-Victor, "Visual Learning by Imitation With Motor Representations," IEEE Transactions on Systems, Man and Cybernetics, Part B (Cybernetics), vol. 35, no. 3, pp. 438-449, 2005.

[5] M. Fadlil, K. Ikeda, K. Abe, T. Nakamura, and T. Nagai, "Integrated concept of objects and human motions based on multi-layered multimodal LDA," in International Conference on Intelligent Robots and Systems. IEEE, 2013, pp. 2256-2263.

[6] T. Araki, T. Nakamura, T. Nagai, K. Funakoshi, M. Nakano, and N. Iwahashi, "Autonomous acquisition of multimodal information for online object concept formation by robots," in International Conference on Intelligent Robots and Systems. IEEE, 2011, pp. 1540-1547.

[7] A. M. Batula, M. Colacot, D. K. Grunberg, and Y. E. Kim, "Using Audio and Haptic Feedback to Detect Errors in Humanoid Musical Performances," International Conference on New Interfaces for Musical Expression, vol. 2, pp. 295-300, 2013.

[8] A. Tidemann, P. Öztürk, and Y. Demiris, "A groovy virtual drumming agent," Lecture Notes in Computer Science, vol. 5773 LNAI, pp. 104$117,2009$.

[9] A. M. Schmidts, D. Lee, and A. Peer, "Imitation learning of human grasping skills from motion and force data," in International Conference on Intelligent Robots and Systems. IEEE, 2011, pp. 1002-1007.

[10] P. Abbeel and A. Y. Ng, "Apprenticeship learning via inverse reinforcement learning," in Twenty-first International Conference on Machine Learning, vol. 69. ACM Internaltional Conference Proceeding Series, 2004, pp. 1-8.

[11] Y. Demiris and A. Dearden, "From motor babbling to hierarchical learning by imitation: a robot developmental pathway," in Proceedings of the 5th International Workshop on Epigenetic Robotics, 2005, pp. 31-37.

[12] K. Mochizuki, S. Nishide, H. G. Okuno, and T. Ogata, "Developmental human-robot imitation learning of drawing with a neuro dynamical system," in International Conference on Systems, Man, and Cybernetics. IEEE, 2013, pp. 2336-2341.

[13] T. Fischer and Y. Demiris, "Markerless perspective taking for humanoid robots in unconstrained environments," in International Conference on Robotics and Automation. IEEE, 2016, pp. 3309-3316.

[14] G. Metta, L. Natale, F. Nori, G. Sandini, D. Vernon, L. Fadiga, C. von Hofsten, K. Rosander, M. Lopes, J. Santos-Victor, A. Bernardino, and L. Montesano, "The iCub humanoid robot: an open-systems platform for research in cognitive development." Neural networks, vol. 23, no. 8-9, pp. 1125-34, 2010.

[15] A. Dearden and Y. Demiris, "Learning forward models for robots," International Joint Conference on Artificial Intelligence, pp. 1440-1445, 2005.

[16] D. M. Wolpert, J. Diedrichsen, and J. R. Flanagan, "Principles of sensorimotor learning." Nature reviews. Neuroscience, vol. 12, no. 12, pp. 739-51, 2011.

[17] S. Hutchinson, G. Hager, and P. Corke, "A tutorial on visual servo control," IEEE Transactions on Robotics and Automation, vol. 12, no. 5, pp. 651-670, 1996.

[18] G. Hager, "A modular system for robust positioning using feedback from stereo vision," IEEE Transactions on Robotics and Automation, vol. 13, no. 4, pp. 582-595, 1997.

[19] D. Berenson, S. S. Srinivasa, D. Ferguson, and J. J. Kuffner, "Manipulation planning on constraint manifolds," in International Conference on Robotics and Automation. IEEE, 2009, pp. 625-632.

[20] P. Kormushev, Y. Demiris, and D. G. Caldwell, "Encoderless position control of a two-link robot manipulator," in International Conference on Robotics and Automation. IEEE, 2015, pp. 943-949.

[21] H. Jin Chang, T. Fischer, M. Petit, M. Zambelli, and Y. Demiris, "Kinematic structure correspondences via hypergraph matching," in Conference on Computer Vision and Pattern Recognition. IEEE, 2016, pp. $4216-4225$. 